\title{
MICROMACHINED BROADSIDE-COUPLED TUNABLE METAMATERIALS
}

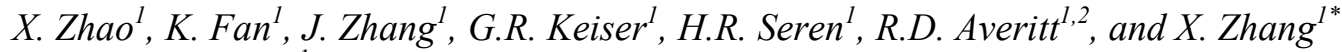 \\ ${ }^{1}$ Boston University, Boston, Massachusetts, USA \\ ${ }^{2}$ University of California, San Diego, La Jolla, California, USA
}

\begin{abstract}
This paper reports a micromachined tunable metamaterial based on broadside-coupled split ring resonators (BC-SRRs). The device consists of two layers of SRRs stacked on top of each other with an air gap spacer, forming the BC-SRRs. One of the layers is fixed, while the other can be driven by an electrostatic comb-drive actuator. The lateral displacement between the two layers of SRRs can be changed by the actuator, resulting in the tuning of resonance frequencies of the BC-SRRs and thus the transmission spectrum of the device. The preliminary results show that the resonance frequency can be tuned up to $100 \mathrm{GHz}$ corresponding to an $18 \mu \mathrm{m}$ lateral displacement. Our tunable metamaterials have promising applications as $\mathrm{THz}$ modulators, filters and sensors.
\end{abstract}

\section{INTRODUCTION}

Metamaterials (MMs) have been considered as a route of engineering the electromagnetic response of micro- and nanostructured materials through resonance excitations [1]. MMs have many remarkable applications, including super lensing [2], negative refractive index [3], cloaking [4] and perfect absorption [5], in security [6, 7], chemical and biology sensing [8], and information processing [9].

In order to enhance the functionalities and practicality of MMs, several approaches have been employed to make the MMs tunable, reconfigurable and dynamic. These include photoexcitation [10], electrical gating [11], thermal tuning [12], adjustment of near-field coupling [13] and structural and mechanical reconfiguration [14]. Among these methods, tuning the near field coupling is of interest because of its high efficiency, stability and ease of controlling [13, 15]. One example of a coupled MM structures is the broadside-coupled split ring resonator (BC-SRR), in which two split ring resonator (SRR) arrays are stacked vertically and rotated $180^{\circ}$ relative to each other. Recent study shows that the lateral, in plane displacement between the two SRR layers will tune the MM resonance frequency significantly without altering the amplitude and Q-factor of the resonance [13]. To our knowledge, however, no BC-SRR based real-time tunable $\mathrm{MM}$ devices have been reported in the literature.

Micromachined electrostatic actuators provide an intuitive path to realize the functional broadside-coupled tunable devices. Comb drive actuation has been successfully used in single layer MM devices to dynamically alter in-plane coupling between MM inclusions and tune the MM's response [14]. However, thick substrate supporting the fixed elements in the MMs, leads to additional insertion loss. Additionally, the translation range of the movable elements is limited by the single layer design of the structure, limiting tunability.

In this paper, we report a broadside-coupled SRR MM at THz frequencies, tunable via comb drive actuation. The $\mathrm{MM}$ is composed of two layered of SRRs, formed BC-SRRs. The first SRR array sits on a thin, moveable silicon layer connected to comb-drive actuators. The second SRR array sits on a fixed silicon nitride film. Notably, the substrate is eliminated in this structure. The MM structure was characterized using the THz Time Domain Spectroscopy at different lateral relative displacement between the SRR layers. The results show that the resonance frequency can be shifted up to $100 \mathrm{GHz}$ corresponding to $18 \mu \mathrm{m}$ lateral displacement.
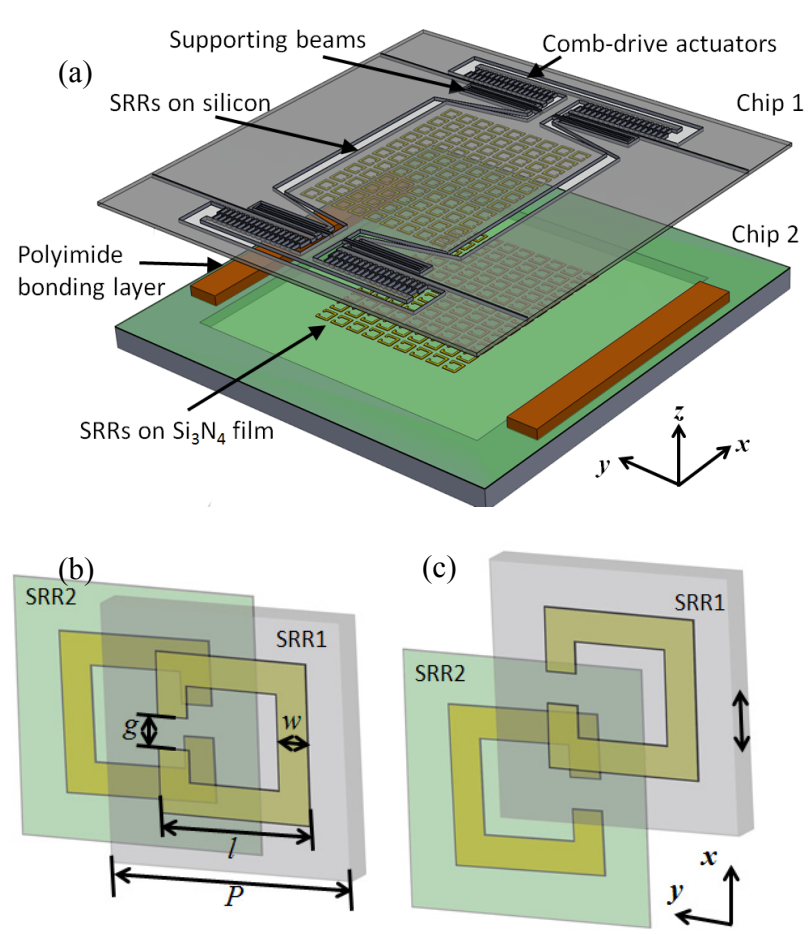

Figure 1: Illustration of the broad-side coupled tunable metamaterials: (a) the exploded diagram of the tunable metamaterials, in which two chips were bonded together with polyimide spacer; (b) and (c) the unit-cell of the MMs with different $x$-axis shift.

\section{DESIGN AND FABRICATION}

The illustration of the designed tunable MMs is shown in Fig.1. The MMs are composed of two layers of SRRs separated by $8 \mu \mathrm{m}$ air gap. One layer is on $10 \mu \mathrm{m}$ thick silicon with electrostatic comb-drive actuators, while the other is fixed on 500nm thick $\mathrm{SiN}_{\mathrm{x}}$ thin film. The unit-cell of the MMs with different $x$-axis displacement is shown in Fig.1 (b) and (c). The rings are rotated $180^{\circ}$ to each other, forming a broadside-coupled configuration. Since the two layers of MMs are closed to each other, the nearfield interaction between them is strong. The relative shift along $x$ axis between the arrays, controlled by the applied voltage, can change the coupling between the SRRs. Thus, it modulates the frequency response of MMs.

The two component SRRs in each unit are not identical because of different substrate materials and designed dimensions as listed in Table 1. Fig. 2 shows the simulated transmission responses of individual SRR under the incident $\mathrm{THz}$ radiation. The resonance frequency of each resonator is determined by the selfinductance and capacitance since $\omega_{0} \sim 1 / \sqrt{ }(L C)$. When they are forming the broadside-coupled pair, as configured in Fig.1 (b), the near field coupling between the rings result in the dual-band response (black curve in Fig.2). According to [13, 16], the coupling arises from the mutual inductance and capacitance. 


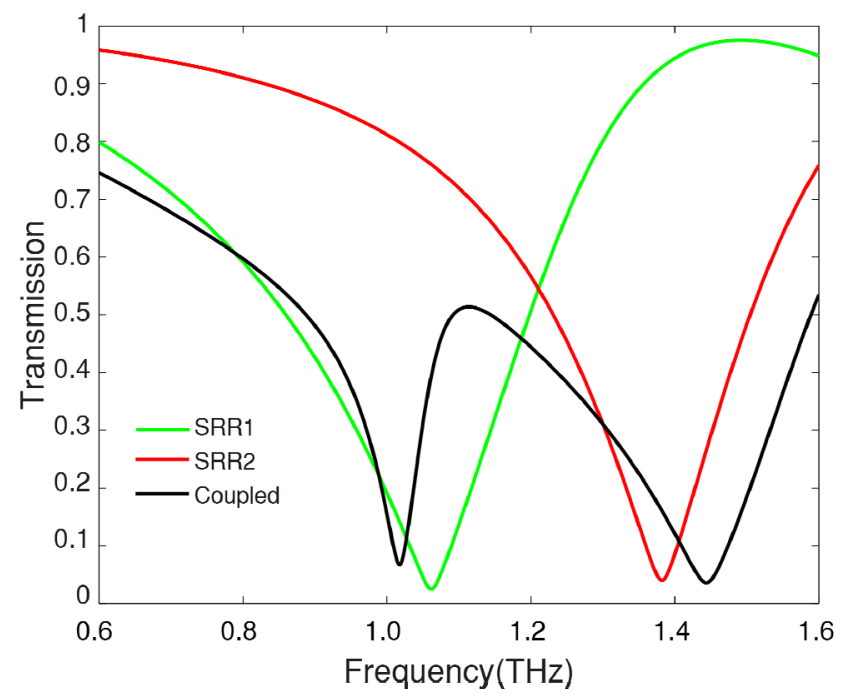

Figure 2: Simulated transmission spectra of the SRRs.

If there is relative lateral displacement between the two layers, the mutual inductance and capacitance will change by reason of the structure reconfiguration. This will in turn alter the transmission spectra.

Fig. 3 shows the fabrication process flow that includes two wafers processing and chip integration. One of the wafers is a silicon-on-insulator (SOI) wafer with $10 \mu \mathrm{m}$ thick device layer, $2 \mu \mathrm{m}$ oxide layer and $500 \mu \mathrm{m}$ handle layer. First, both sides of the SOI wafer were coated by $500 \mathrm{~nm} \mathrm{SiN}_{\mathrm{x}}$ film with LPCVD, followed by patterning the top side with RIE. Next, the Al electrodes were patterned by e-beam evaporation and lift-off, followed by rapid thermal annealing at $450^{\circ} \mathrm{C}$ in $\mathrm{H}_{2} / \mathrm{N}_{2}$ forming gas for $30 \mathrm{~min}$ to realize the Ohmic contact. The $150 \mathrm{~nm}$ thick gold SRRs were also patterned by lift-off process after that. Then, the device layer of SOI wafer was etched to realize the comb finger and beam structure using DRIE. Lastly, the handle layer of silicon was etched through by DRIE and the buried oxide layer was etched by Silox Vapox III etchant (Transene Inc.) to release the structure. The second wafer is a double side polished silicon wafer. It was coated by $\mathrm{SiN}_{\mathrm{x}}$ and the SRRs were patterned firstly. Afterward, etching windows were patterned on the backside $\mathrm{SiN}_{\mathrm{x}}$ by RIE and the wafer was soaked in $\mathrm{KOH}$ solution until a free-standing $\mathrm{SiN}_{\mathrm{x}}$ thin film. Both wafers were diced into chips after these processes. Finally, an $8 \mu \mathrm{m}$ thick layer of photo-definable polymer was patterned to bond the two chips with flip chip bonder.

Fig. 4 is the microscopic image of a chip with comb-drive actuator. Two identical actuators driven by electrostatic force were placed on both sides of the MMs array. The actuators on each side provide unidirectional in-plane translation along $x$-axis. The movable structure is suspended by four pairs of folded beam. The actuation relationship $\Delta x=A V^{2}$, where $\Delta x$ is the displacement, $V$ is the actuation voltage and $A$ is the actuation coefficient. In our structure, the actuation coefficient of the structure is $A=5 \mathrm{~nm} / \mathrm{V}^{2}$, which is verified by experiment. This coefficient can be further improved by fabricating smaller gaps between fingers. Fig. 5 shows the fabricated device. The two chips were bonded together with polyimide spacer. The chip is wire bonded to a customized PCB for the electric connection. Fig.5 (d) and (e) are showing the SRRs position without displacement and with $18 \mu \mathrm{m}$ displacement.
Table 1: The dimensions of SRRs (unit in $\mu \mathrm{m}$ )

\begin{tabular}{|c|c|c|c|c|}
\hline & $\begin{array}{c}\text { Periodicity } \\
(P)\end{array}$ & $\begin{array}{c}\text { Side-width } \\
(l)\end{array}$ & $\begin{array}{c}\text { Linewdith } \\
(w)\end{array}$ & $\begin{array}{c}\text { Gap } \\
(g)\end{array}$ \\
\hline SRR1 & 58 & 40 & 10 & 14 \\
\hline SRR2 & 58 & 40 & 10 & 16 \\
\hline
\end{tabular}

(a)

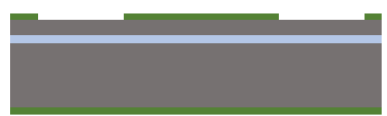

(b)

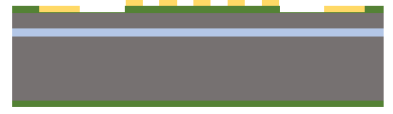

(c)

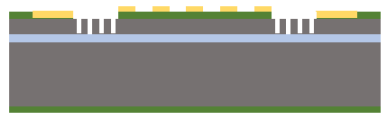

(d)
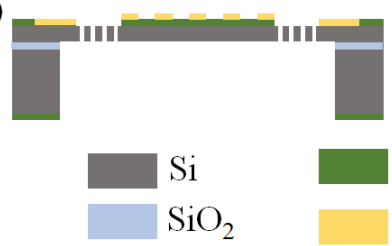

(e)

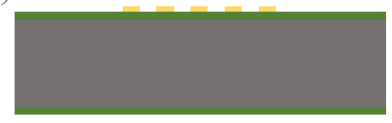

(f)

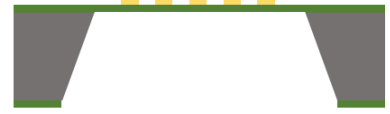

(g)

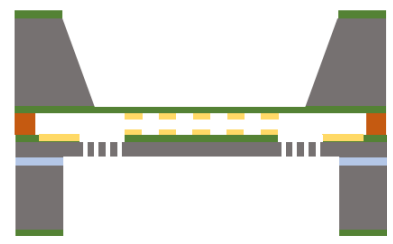

$\mathrm{SiN}_{\mathrm{x}}$

Polyimide
Figure 3: Fabrication process of the tunable metamaterials. (a)-(d) the first (SOI) wafer process, (e)-(f) the second (silicon) wafer process, (g) the flip-chip bonding.
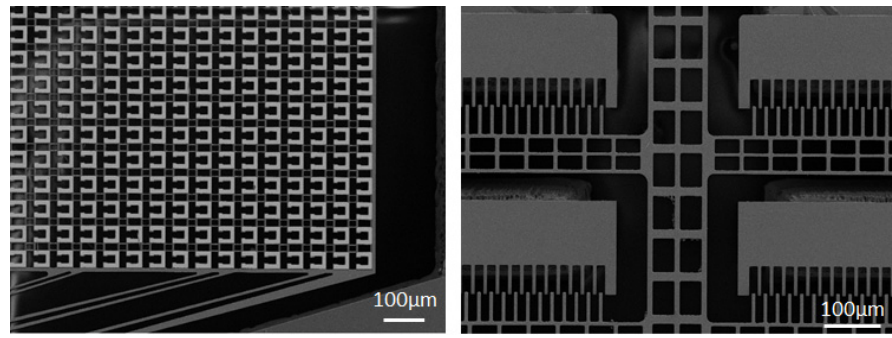

Figure 4: The microscopic image of the chip with comb-drive actuator.

\section{EXPERIMENT AND RESULTS}

The fabricated device was characterized by Terahertz Time Domain Spectroscopy (THz-TDS) system [12]. A THz pulse with diameter of $3 \mathrm{~mm}$ was normally incident on the MM surface with electric component polarized parallel to the sides of SRRs with capacitive gaps. The transmission signal through the tunable MMs was measured at different driving voltage applied to the combdrive actuator. Another comb-drive structure without SRRs on it was used as reference. The measured transmission spectra are shown in Fig.6. When the lateral shift is $0 \mu \mathrm{m}$ [as shown in Fig.5 (d)], the two SRRs in each unit are not well-aligned in $x$-axis. There are two resonance modes observed at $1.1 \mathrm{THz}$ and $1.4 \mathrm{THz}$. Upon increasing the lateral displacement, the relative position as shown in Fig.5 (e). The coupling between the two SRRs in each unit increases in this configuration. This increase in coupling strength causes a mode splitting redshifting the lower resonance by 

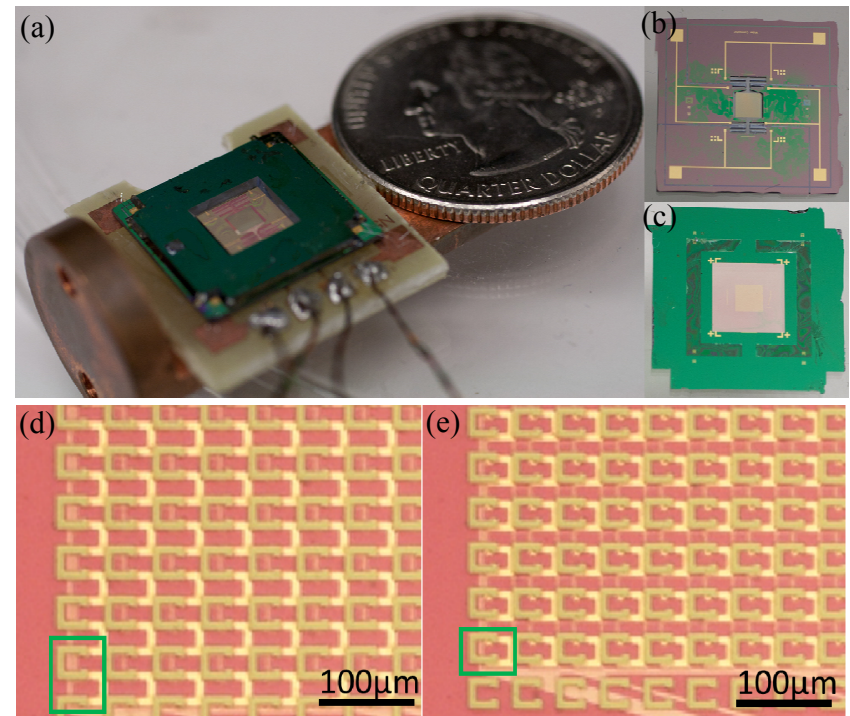

Figure 5: The fabricated deivces. (a) The bonded chip wire bonded on a printed circuit board; (b) the SOI chip (chip 1); (c)

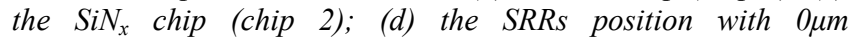
displacement; (e) the SRRs position with $18 \mu \mathrm{m}$ displacement.

$50 \mathrm{GHz}$ and blueshifting the higher resonance by $100 \mathrm{GHz}$ for a lateral displacement of $18 \mu \mathrm{m}$.

\section{DISCUSSION}

Multiple methods exist to model the coupling behavior of broadside-coupled SRRs [17], including coupled mode theory, mode hybridization model and mutual capacitance and inductance model [18]. Herein, we will follow the third method to describe the tuning mechanism in our device.

In order to have better understanding of the coupling mechanism at work in this structure, we performed finite difference analysis on the device with CST Microwave studio. The results are shown in Fig.7. When the lateral shift is $0 \mu \mathrm{m}$, the current oscillates only in SRR1 at the lower resonance frequency [Fig.7 (b)] and only in SRR2 at higher resonance frequency [Fig.7 (c)]. This suggests that the mutual capacitance and inductance are negligible and the two resonators are resonating independently. For the case of maximum lateral shift, the configuration of the two layers of SRRs in Fig.5 (e), both of the SRRs are excited simultaneously at both resonance modes. At the first mode, the currents in the two SRRs are in phase [Fig.7 (d)] and generate outof-plane magnetic fields pointing the same direction. Hence, the mutual inductance at this mode is positive, which means it can increase the total inductance of the system. At the second mode, the currents are oscillating out-of-phase [Fig.7 (e)], which will induce magnetic field in the opposite directions. As a result, the mutual inductance is negative and will decrease the total inductance. The mutual capacitance is almost negligible because of the small overlap area between the SRRs. Thus, lateral displacement increases the absolute value of the mutual inductance. Consequently, the total inductance increases and causes a redshift in the first mode but decreases and causes a blueshift in the second mode. The simulated transmission spectra shown in Fig.7 (a) agree well with the experimental results, lending support to this theoretical model.

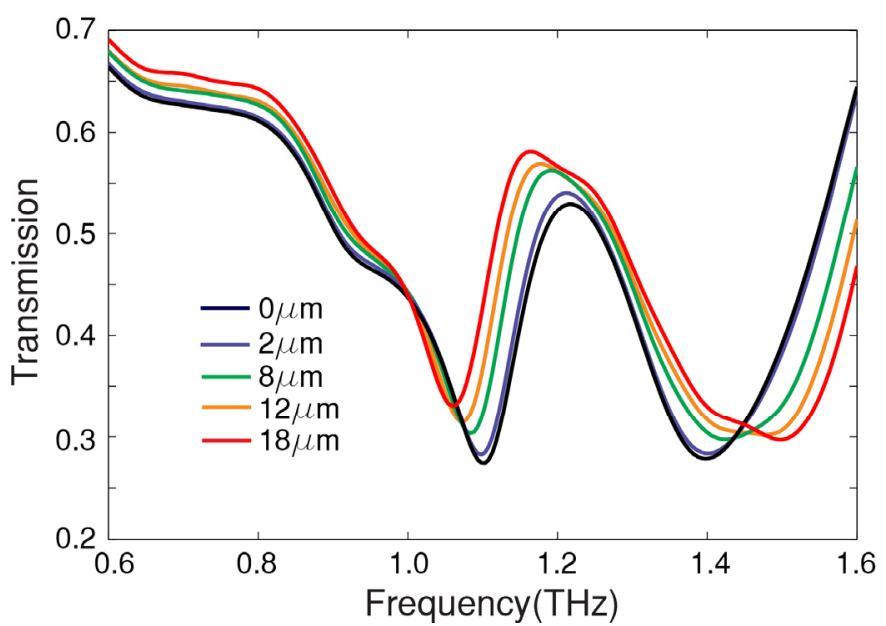

Figure 6: The experimental transmission spectra of the tunable metamaterials at different lateral shift.

(a)
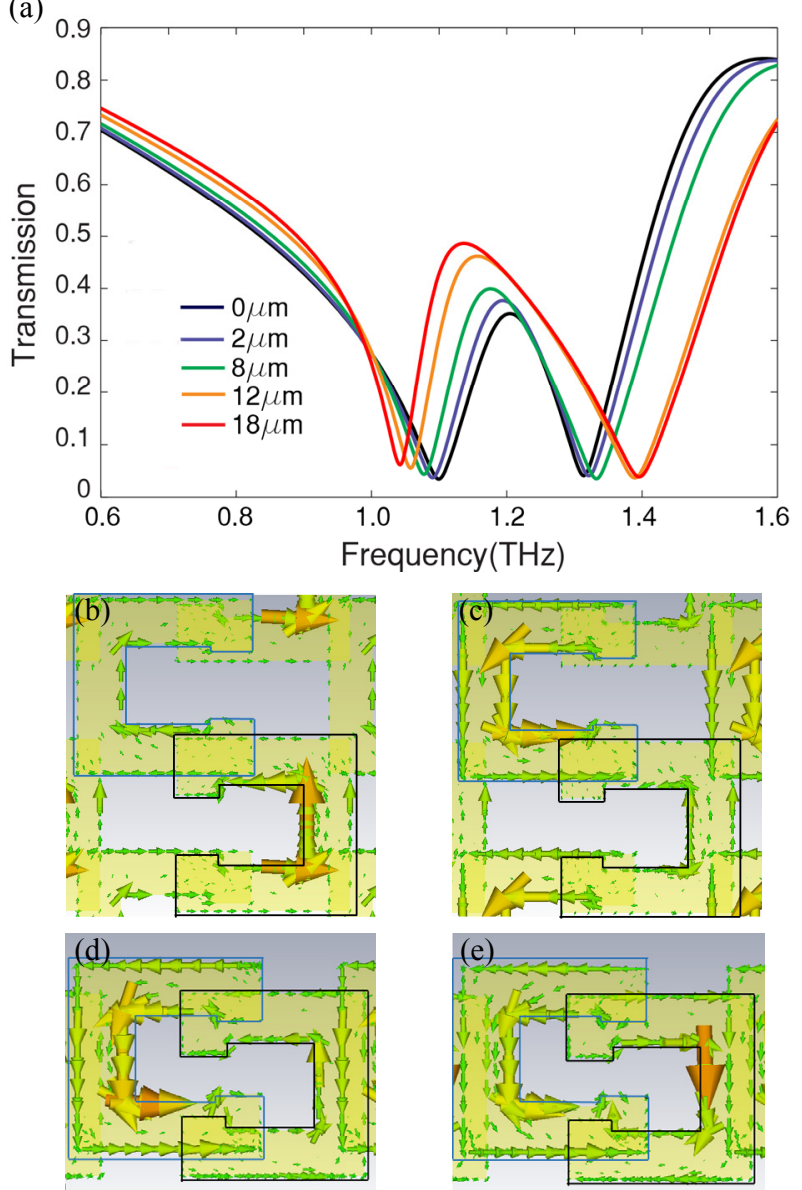

Figure 7: (a) The simulated transmission spectra of the tunable metamaterials at different relative displacement; (b) and (c) the current distribution at $1.10 \mathrm{THz}$ and $1.30 \mathrm{THz}$ with $0 \mu \mathrm{m}$ displacement; (d) and (e) the current distribution at $1.03 \mathrm{THz}$ and $1.40 T H z$ with $18 \mu \mathrm{m}$ displacement (The SRR in black frame is SRR1 while the blue SRR2). 


\section{CONCLUSIONS}

A real-time tunable dual-band MM based on broadsidecoupled SRRs with a micromachined actuator was designed, fabricated and characterized. The resonance frequency redshifts $50 \mathrm{GHz}$ at the first mode and blueshifts $100 \mathrm{GHz}$ at the second mode with $18 \mu \mathrm{m}$ lateral shift. The nature of the frequency shift was studied qualitatively by mutual inductance model. Further optimization of this device will lead to an increased effective frequency scanning range. This $\mathrm{MM}$ device has potential applications for spectral sensing of chemicals and dynamic modulation of $\mathrm{THz}$ radiation.

\section{ACKNOWLEDGEMENT}

This work was supported in part by the National Science Foundation under Contract ECCS 1309835, the Air Force Office of Scientific Research under Contract FA9550-09-1-0708, and DTRA under Contract W911NF-06-2-0040 administered by the Army Research Laboratory. The authors would like to thank Boston University Photonics Center for technical support. Travel support has been provided by Transducer Research Foundation.

\section{REFERENCES}

[1] N.I. Zheludev and Y.S. Kivshar, "From Metamaterials to Metadevices", Nature Materials, 11, 917 (2012).

[2] X. Zhang, and Z. Liu, "Superlenses to Overcome the Diffraction Limit", Nature Materials, 7, 435 (2008).

[3] D.R. Smith, J.B. Pendry, and M.C. Wiltshire, "Metamaterials and Negative Refractive Index", Science, 305, 788 (2004).

[4] D. Schurig, J.J. Mock, B.J. Justice, S.A. Cummer, J.B. Pendry, A.F. Starr, and D.R. Smith, "Metamaterial Electromagnetic Cloaking at Microwave Frequencies", Science, 314, 977 (2006).

[5] N.I. Landy, S. Sajuyigbe, J.J. Mock, D.R. Smith, and W.J. Padilla, "Perfect Metamaterial Absorber", Physical Review Letters, 100, 207402 (2006).

[6] L. Feng, Y. Xu, W.S. Fegadolli, M. Lu, J.E. Oliveira, V.R. Almeida, Y. Chen, and A. Scherer, "Experimental Demonstration of a Unidirectional Reflectionless Parity-time Metamaterial at Optical Frequencies", Nature Materials, 12, 108 (2012).

[7] D. Shrekenhamer, C.M. Watts, J. Montoya, S. Krishna, and W.J. Padilla, "Metamaterial-based Imaging for Potential Security Applications", Proceeding of SPIE, 8632, 863321 (2013).
[8] X. Xu, B. Deng, D. Li, J. Zhang, L. Wong, Q. Zhang, S Wang, and Q. Xiong, "Flexible Visible-Infrared Metamaterials and Their Applications in Highly Sensitive Chemical and Biological Sensing”, Nano Letters, 11, 3232 (2011).

[9] N. Engheta, "Circuits with Light at Nanoscales: Optical Nanocircuits Inspired by Metamaterials", Science, 317, 1698 (2007).

[10] K. Fan, X. Zhao, J. Zhang, K. Geng, G.R. Keiser, H.R. Seren, G.D. Metcalfe, M. Wraback, X. Zhang, and R.D. Averitt, "Optically Tunable Terahertz Metamaterials on Highly Flexible Substrates", IEEE Transcation on Terahertz Science and Technology, 3, 702 (2013).

[11] H.-T. Chen, W.J. Padilla, M.J. Cich, A.K. Azad, R.D. Averitt, and A.J. Taylor, "A Metamaterials Solid-State Terahertz Phase Modulator", Nature Photon, 3, 148 (2009).

[12] H. Tao, A.C. Strikwerda, K. Fan, W.J. Padilla, X. Zhang, and R.D. Averitt, "MEMS Based Structurally Tunable Metamaterials at Terahertz Frequencies", Journal of Infrared, Milimeter and Terahertz Waves, 32, 580 (2011).

[13] E. Ekmekci, A.C. Strikwerda, K. Fan, G.R. Keiser, X. Zhang, G. Turhan-Sayan, and R.D. Averitt, "Frequency Tunable Terahertz Metamaterials Using Broadside Coupled Split-ring Resonators", Physical Review B, 83, 193103 (2011).

[14] A.Q. Liu, W.M. Zhu, D.P. Tsai, and N.I. Zheludev, "Micromachined Tunable Metamaterials: A Review", Journal of Optics, 14, 114009 (2011).

[15] A.D. Boardman, V.V. Grimalsky, Y.S. Kivshar, M. Lapine, and N. Litchinister, "Active and Tunable Metamaterials", Laser Photonics Review, 5, 287 (2011).

[16] G.R. Keiser, A.C. Strikwerda, K. Fan, V. Young, X. Zhang, and R.D. Averitt, "Decoupling Crossover in Asymmetric Broadside Coupled Split-Ring Resonators at Terahertz Frequencies", Physical Review B, 88, 023101 (2013).

[17] H. Haus, and W.P. Huang, "Couple-Mode Theory", Proceedings of the IEEE, 79, 10 (1991).

[18] G.R, Keiser, K. Fan, X. Zhang, and R.D. Averitt, "Towards Dynamic, Tunable, and Nonlinear Metamaterials via Near Field Interactions: A Review", Journal of Infrared, Milimeter and Terahertz Waves, 34, 709 (2013).

\section{CONTACT}

* X. Zhang, tel: + 1.617.358.2702; xinz@bu.edu 